\title{
Visceral plexiform schwannoma: A case series
}

\author{
FRANCESCA DESTRO $^{1}$, SHILPA SHARMA ${ }^{2}$, LUCIANO MAESTRI ${ }^{1}$, \\ CLAUDIO VELLA $^{1}$, PAOLA COLLINI ${ }^{3}$ and GIOVANNA RICCIPETITONI ${ }^{1}$
}

\author{
${ }^{1}$ Department of Paediatric Surgery, Buzzi Children's Hospital, I-20154 Milan, Italy; ${ }^{2}$ Department of Paediatric Surgery, \\ All India Institute of Medical Sciences, New Delhi 110029, India; ${ }^{3}$ Department of Diagnostic Pathology \\ and Laboratory Medicine, Fondazione IRCCS Istituto Nazionale dei Tumori, I-20133 Milan, Italy
}

Received December 9, 2019; Accepted October 10, 2020

DOI: $10.3892 / \mathrm{mco} .2020 .2176$

\begin{abstract}
Plexiform schwannoma (PS) is a benign tumour of the peripheral nerve sheath that is typically found in the skin. Fewer than 15 cases of visceral PS have been reported to date in both adults and children. We herein discuss a series of 3 patients ( 2 male and 1 female) with abdominal PS, aged 10-16 years (mean age, 12 years). All the patients had an acute presentation with abdominal pain, which was associated with rectal bleeding in 1 case and with walking difficulties in 1 case. Radiological investigations included abdominal magnetic resonance imaging (MRI) and computed tomography, along with neurofibromatosis screening (cerebral MRI and dermatological evaluation). Complete removal of the mass was possible in 2 of the patients (in 1 case by laparoscopically assisted surgery). Follow-up was uneventful. Abdominal PS is a rare occurrence. Due to its possible association with neurofibromatosis, the diagnosis of PS should prompt an investigation for other manifestations of this disorder.
\end{abstract}

\section{Introduction}

Plexiform schwannoma (PS) is a benign tumour of the peripheral nerve sheath, in which Schwann cells exhibit a multinodular growth pattern (plexiform) (1). PS represents a unique variant, accounting for $\sim 5 \%$ of all schwannomas (2). Berg et al (3) reported a series of 2,259 schwannomas and found that PS represented only $4.3 \%$ (97 patients) of the total cases.

PS is typically found in the skin, while visceral localization is rare $(2,4)$. The series from the Mayo Clinic described 3 patients (3/97, 3\%) with visceral PS, involving the lung, sigmoid colon and parathyroid gland (3). To date, there have been fewer than 15 reported cases of intra-abdominal and

Correspondence to: Dr Francesca Destro, Department of Paediatric Surgery, Buzzi Children's Hospital, 32 Via Castelvetro, I-20154 Milan, Italy

E-mail: francesca.destro@asst-fbf-sacco.it

Key words: plexiform schwannoma, children, abdominal pain, rectal bleeding visceral PS in both adults and children $(3,5)$. In particular, mesenteric PS has never been reported, to the best of our knowledge.

PS is typically characterised by a benign course, but it may be associated with neurofibromatosis (NF) and the differential diagnosis from its malignant counterparts (e.g., neurofibroma and malignant peripheral nerve sheath tumour) may be difficult (6). The aim of the present study was to retrospectively describe two unique cases of PS with a visceral location in young children, as well as one case that has been previously reported by one of the authors, in order to highlight the importance of considering this entity in the differential diagnosis of abdominal masses and to perform a careful investigation of associated abnormalities, particularly NF.

\section{Case reports}

Case 1. A 16-year-old female patient was admitted to Buzzi Children's Hospital in July 2017 due to acute onset of abdominal and back pain associated with fever and rectal bleeding. Over the last few months, the patient had suffered from recurrent episodes of abdominal pain that were attributed to constipation and treated with stool softeners. The family history was unremarkable.

Upon admission, the patient was in good clinical condition and the results of the laboratory tests were unremarkable.

On abdominal ultrasound (Fig. 1), an epigastric mass sized $3.5 \mathrm{~cm}$ was identified. Magnetic resonance imaging (MRI) examination (Fig. 2) confirmed the presence of an irregular solid inhomogeneous mass in the left upper abdomen with contrast enhancement. The Tc-99m-octreotide scintigraphy was negative.

During laparoscopy (Fig. 3), a hard polylobulated mesenteric mass was identified close to the first jejunal loops. The mass and the affected bowel loops were exteriorized through the umbilical wound and resected along with the adjacent intestine (total length, $10 \mathrm{~cm}$ ). Intestinal continuity was restored by termino-terminal anastomosis and the mesenteric gap was closed.

Histological examination (Fig. 4) following hematoxylin and eosin staining revealed a PS with free margins. Immunohistochemical staining for S100, CD34, epithelial membrane antigen (EMA) and $\alpha$-smooth muscle actin ( $\alpha$-SMA) was also performed (negative $\alpha$-SMA and CD34; positive S100 and EMA). The screening for NF, including dermatological evaluation and cerebral MRI, was normal. 
Two years after the surgery the patient remained asymptomatic, and an abdominal MRI revealed no abnormalities (last follow-up visit, December 2019).

Case 2. A 10-year-old boy was referred to New Delhi All India Institute of Medical Sciences in May 2013 with gross abdominal distension and pain. On palpation, there was a lump in the abdomen. The computed tomography scan revealed a mass with a whorled appearance. On exploratory laparotomy, a sizeable mass (greatest diameter, $14 \mathrm{~cm}$ ) arising from the retroperitoneum and engulfing the aorta and inferior vena cava was identified. Frozen section biopsy revealed a benign lesion. Near-total excision of the mass was performed, shaving it off but leaving a part attached to the great vessels. Histopathological examination was suggestive of PS. The immunohistochemical staining for S100 was positive. Screening for NF was performed, including dermatological evaluation and cerebral and cervical MRI, and the findings were normal. The patient was asymptomatic after 4 years of follow-up (last follow-up visit, January 2018).

Case 3. This case was previously reported by one of the authors (7). In April 2003, a presacral schwannoma with intraspinal extension was diagnosed in a 10 -year-old boy with type $2 \mathrm{NF}$ and multiple neurofibromas, who was admitted to the New Delhi All India Institute of Medical Sciences with left sciatic pain and difficulty walking. MRI examination revealed a unilateral vestibular schwannoma associated with an intrathoracic schwannoma. Surgery was completed in two steps: The intraspinal portion of the mass was first removed, followed by removal of the presacral portion after 15 days, achieving complete removal. Histological examination revealed S-100 positivity. Our experience with this case enabled us to correctly diagnose case 2 . The patient was asymptomatic and disease-free at the last follow-up visit (February 2008).

\section{Discussion}

In 1978, Harkin et al first described six cases of PS, a rare Schwann cell tumour variant (4.3-5\% of all schwannomas) with intraneural, plexiform and possible multinodular growth (1).

PS is frequently located in superficial soft tissues or the cutaneous region (3). Visceral involvement is extremely rare (3\%) and only a few cases involving the abdomen/digestive tract have been reported to date $(2,8)$. The first case of visceral PS (ascending colon) was reported by Hirose et al in 1997 (9).

In 2016, Kudose et al identified 10 cases of PS arising in the digestive tract ( 5 in the colon, 3 in the oesophagus and 2 in the small intestine) in a review of the English medical literature and reported the first case of gastric PS associated with NF2 (2). Only 2 patients were aged $\leq 18$ years (age range, 11-77 years). The majority of the patients $(6 / 10$ cases) presented with acute symptoms, including abdominal pain and rectal bleeding, as the patients in the present study.

To the best of our knowledge, case 1 presented herein is the first reported case of a mesenteric PS.

PS is generally associated with benign characteristics, slow growth, and no metastatic potential. It usually affects young adults and presents as a solitary lesion $(2,7)$.

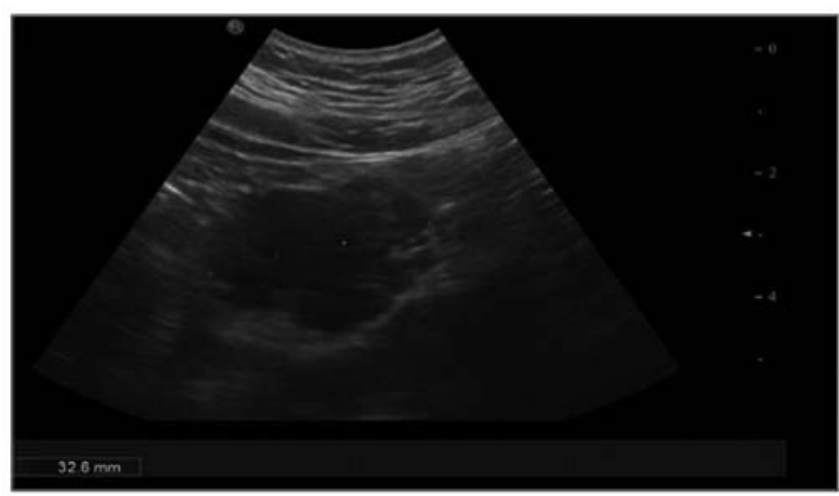

Figure 1. The abdominal ultrasound examination showing an irregular abdominal mass, sized $3.5 \mathrm{~cm}$, in the epigastric region, resembling enlarged lymph nodes.

On histological evaluation, PS has several elements found in conventional schwannoma, such as being composed by compact Schwann cells (but arranged as multinodular/plexiform rather than in a globular configuration), Verocay bodies, hyalinized and/or ectatic vessels and collagenous residues (3). Recent studies also demonstrated that PS may not always display the Antoni A and B patterns (but when it does, Antoni $A$ is predominant) and is often positive for $\mathrm{S} 100$ expression (3-5). A precise definition of the histopathological characteristics is extremely important, as it is the best method for differentiating PS from other benign or malignant lesions. Indeed, preoperative diagnosis is extremely difficult, as the endoscopic and radiological findings are non-specific (5).

On imaging evaluation of case 1 , a mass that was isointense relative to skeletal muscle was identified on MRI T1-weighted images, displaying multinodularity, as previously reported $(2,4,7,10)$, but this was not enough to confirm the diagnosis. A preoperative or intraoperative biopsy was considered; however, the mass was well-defined and appeared to be benign macroscopically. Moreover, the resection also included the normal adjacent bowel, with a total length of $10 \mathrm{~cm}$ of intestine removed. As there is the possibility of other neuronal tumours with different natural histories and behaviours, a preoperative or intraoperative biopsy should be considered to optimise the treatment approach (11). The differential diagnosis from other entities, such as plexiform neurinoma, which is associated with a high risk of malignant transformation, and malignant cell tumours, is crucial in order to avoid delay in treatment and, potentially, the need for a more aggressive approach (7).

Another important aspect is the possible association of PS with NF2 and schwannomatosis that has been reported in up to $10 \%$ of cases $(5,9)$. The presence of multiple lesions represents a high risk of having NF2 and is associated with mutations of the NF2 gene on chromosome 22q12.2 (adjacent to the SMARCB1/INI1 suppressor gene) (12). Therefore, it is important to exclude the presence of associated dermal lesions and neurinoma in patients with PS, particularly in paediatric patients in whom inherited syndromes are more frequent, by performing thorough intracranial and spinal MRI examinations $(11,13)$. Genetic studies are useful when there is a positive family history or in case of a certain association with NF-schwannomatosis. In these selected patients, attempts 

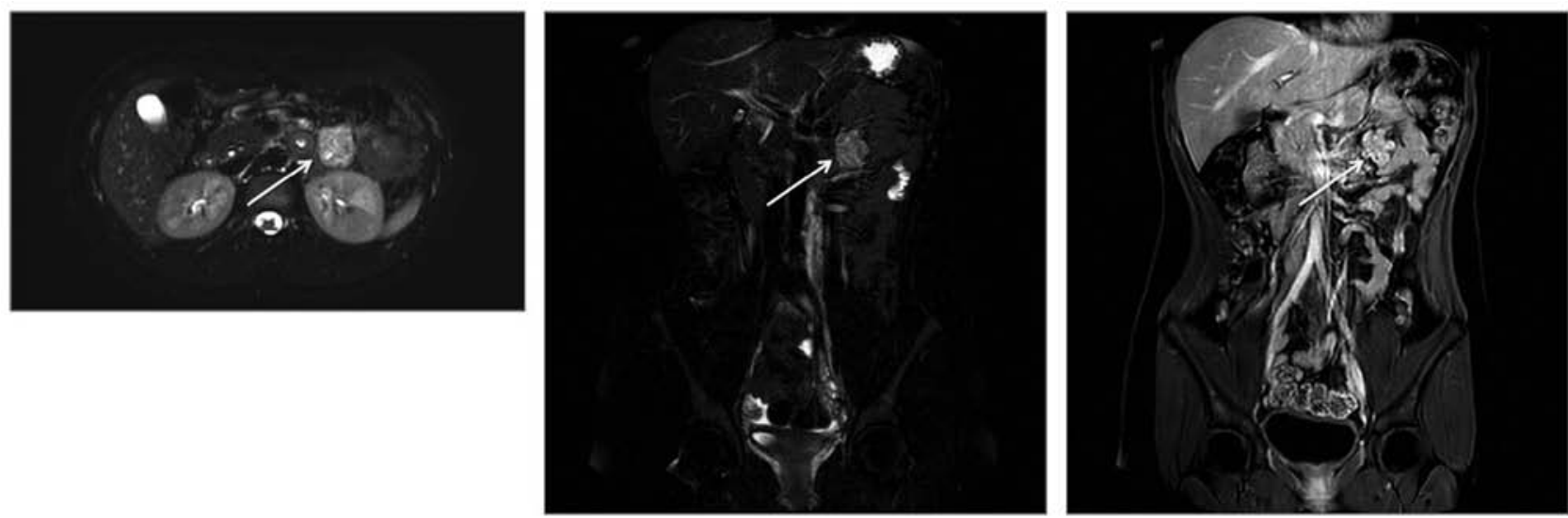

Figure 2. The abdominal magnetic resonance imaging examination confirmed the presence of an irregular solid mass (37x29x32 mm) between the jejunal loops with contrast enhancement (arrows). The mass was isointense relative to adipose tissue on T2-weighted images and iso-hypointense relative to skeletal muscle on T1-weighted images.
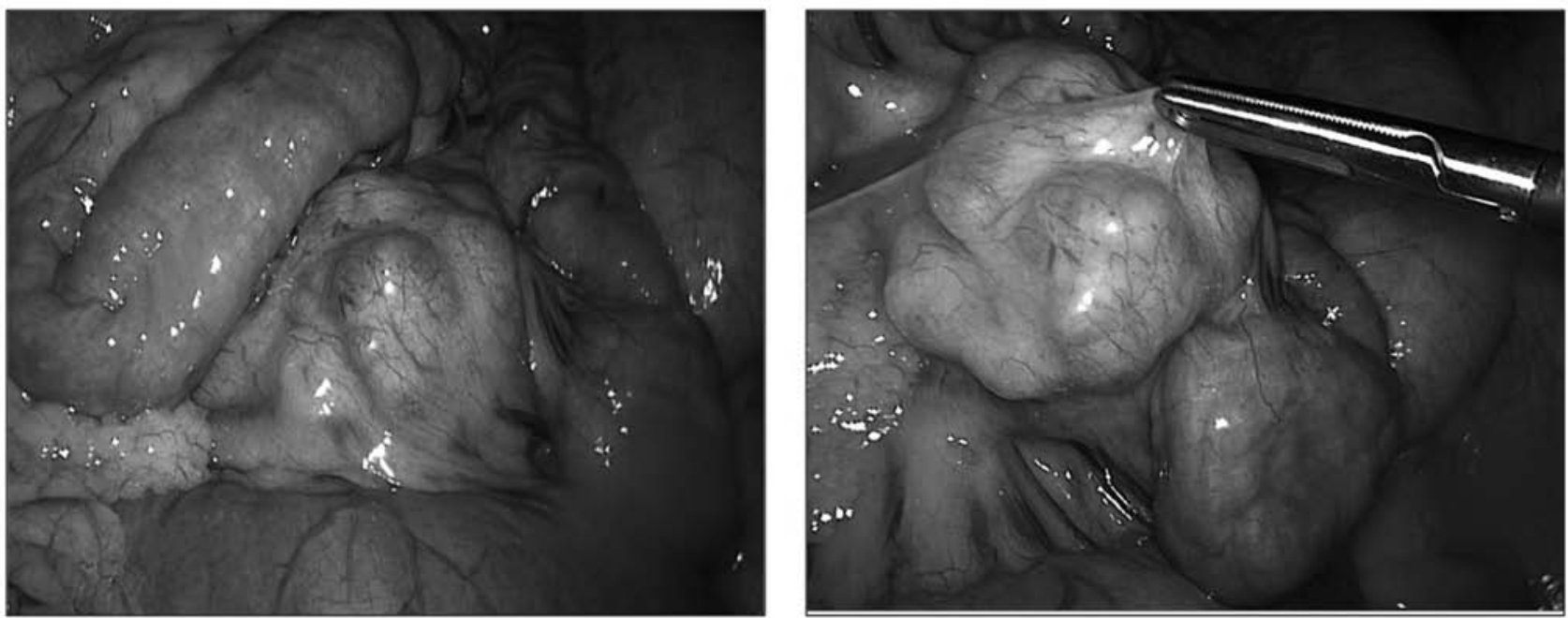

Figure 3. Intraoperatively, a hard polylobulated mesenteric mass was identified close to the first jejunal loops.

have been made to use DNA next-generation sequencing to detect copy number alterations and mosaicisms and to correlate genotype with clinical phenotype (14). This may lead to a better understanding of the underlying pathology and to the development of new clinical models (e.g., germline mutations of SMARCB1 predispose to schwannomas) (15). Moreover, genetic studies are the base of new therapeutic strategies, such as the inhibition of the tyrosine kinase receptor MET or EGFR/ErbB2 and FAK1 (PTK2) to suppress tumorigenesis and achieve a growth-inhibitory effect (16). It is unclear whether there is a correlation between these aspects and the isolated form of PS; SMARCB1/INI1 immunoreactivity appears to be uninformative, particularly in solitary schwannomas (17). On the other hand, recent studies focused on the possibility to perform histological and genetic characterization of Schwann cells from cutaneous PS, correlating them to the presence of NF2 and allowing an early diagnosis of the associated syndrome (18). The same concept may be applied to cells obtained from visceral PS.

PS is treated with radical surgical excision to prevent local recurrence (19). The literature also reports endoscopic submucosal resection in case of rectal PS confined to the submucosa $(4,20)$. Radical excision is associated with an excellent outcome; however, if the mass involves vital structures, it may be possible to perform limited resection. Indeed, the tumour in case 2 engulfed the great vessels in the abdomen; once the diagnosis was confirmed by frozen section biopsies, partial removal was deemed a safer option. Our choice of treatment was also supported by the reported absence of malignant characteristics and metastatic spread of visceral PS (21). In 2017, a series of paediatric non-vestibular schwannoma cases indicated the presence of $>4$ mitoses/10 high-power fields as a risk factor for recurrence (17). Unfortunately, the rare visceral location of PS prevents drawing definitive conclusions regarding partial surgical removal.

Retrosi et al suggest collecting data from patients with PS in unusual locations to advance the overall knowledge and plan adequate management and follow-up (11). Planning follow-up represents another uncertain and critical aspect of patient management. According to Kawaguchi et al, surveillance is not necessary following complete surgical resection (5), but there are no specific indications in case of incomplete removal. 
A
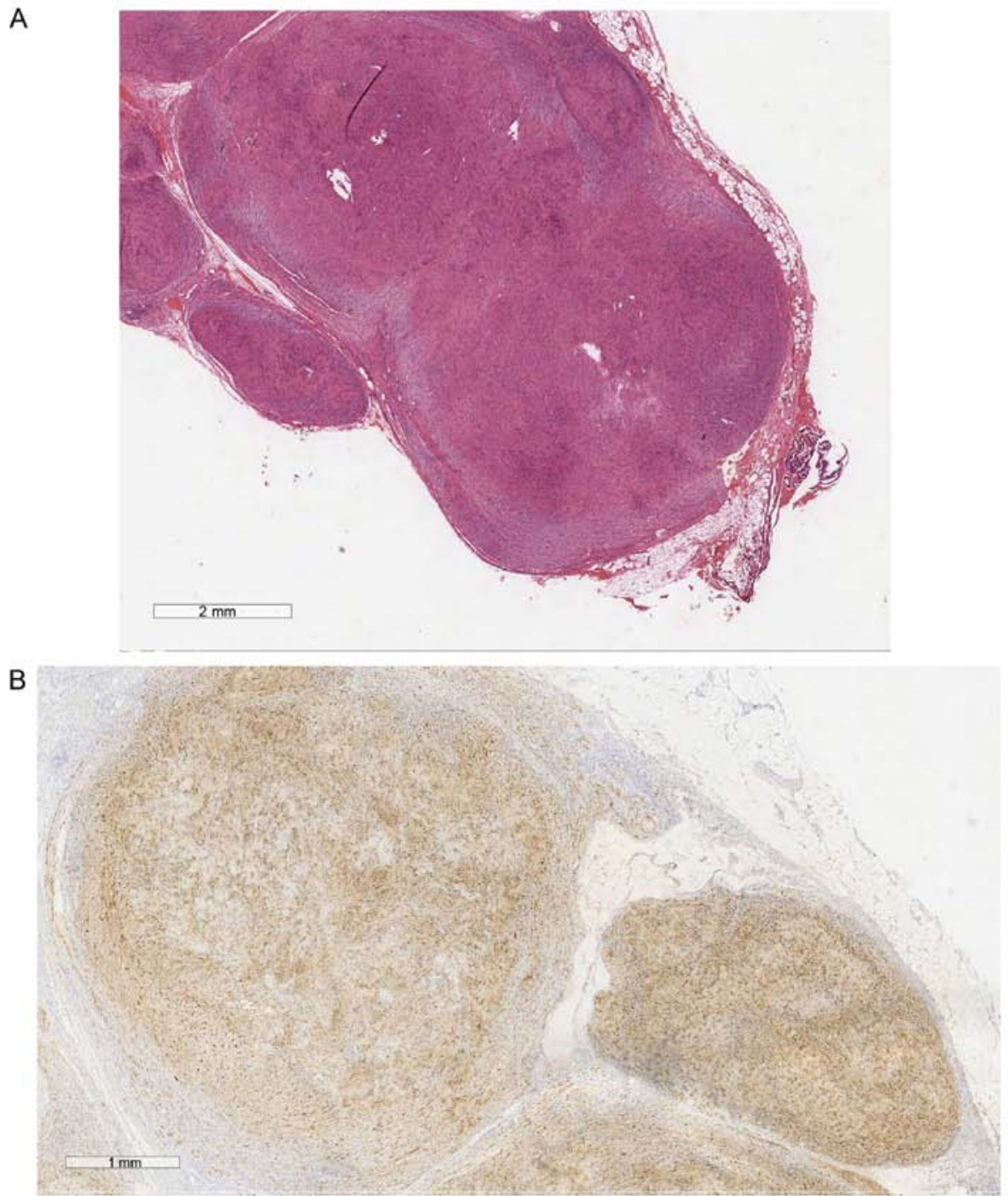

C

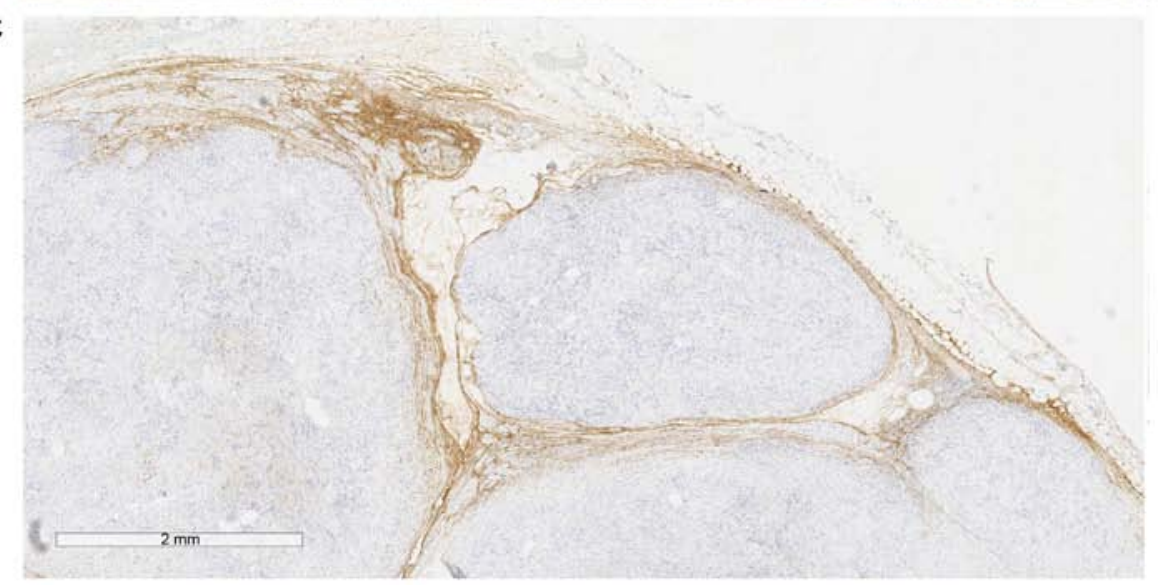

Figure 4. (A) Low-power appearance of the distinctive growth pattern of the tumour following haematoxylin and eosin staining (magnification, $\mathrm{x} 1$; scale bar, $2 \mathrm{~mm}$ ). (B) The tumour was composed of multiple discontiguous nodules of S100-positive Schwann cells with the typical characteristics of Schwannoma (magnification, $x 2$ scale bar, $1 \mathrm{~mm}$ ), (C) encircled by an epithelial membrane antigen-positive perineural capsule (magnification, $\mathrm{x} 4$; scale bar, $2 \mathrm{~mm})$.

Considering the cases reported in the literature as well as our experience, it appears that the histological and biological characteristics of the tumour affect its natural history more than its location: PS has a benign nature, regardless of its localisation. The difficulties in preoperative diagnosis and the asymptomatic pattern in younger patients may explain the relatively late identification and diagnosis of visceral and abdominal PS. However, this does not appear to affect postoperative outcomes, particularly when complete surgical excision is achieved.

In conclusion, although benign schwannomas rarely occur in the gastrointestinal tract, PS should be considered in the 
differential diagnosis of all patients with an abdominal mass As the lesion is benign, subtotal resection may be a viable option if the tumour involves vital structures.

Given its possible association with NF, the diagnosis of PS should prompt an investigation for other manifestations of this disorder.

\section{Acknowledgements}

Not applicable.

\section{Funding}

No funding was received.

\section{Availability of data and materials}

The datasets used and/or analyzed during the present study are available from the corresponding author on reasonable request.

\section{Authors' contributions}

FD and SS conceptualized and co-wrote the manuscript. FD and LM performed the literature search. LM was actively involved in drafting the manuscript. PC performed the histological examination and provided the related image. $\mathrm{CV}$ and GR acted as supervisors and critically revised the manuscript for important intellectual content. All the authors have read and approved the final version of the manuscript.

\section{Ethics approval and consent to participate}

This article does not contain any studies with human participants performed by any of the authors.

\section{Patient consent for publication}

Verbal (case 1) and/or written (cases 2 and 3) informed consent was obtained from all individual participants included in the study or their parents/legal guardians.

\section{Competing interests}

The authors declare that they have no competing interests.

\section{References}

1. Harkin JC, Arrington JH and Reed RJ: Benign plexiform schwannoma, a lesion distinct from plexiform neurofibroma (abstract). J Neuropathol Exp Neurol 37: 622, 1978.

2. Kudose S, Kyriakos M and Awad MM: Gastric plexiform schwannoma in association with neurofibromatosis type 2. Clin J Gastroenterol 9: 352-357, 2016.

3. Berg JC, Scheithauer BW, Spinner RJ, Allen CM and Koutlas IG: Plexiform schwannoma: A clinicopathologic overview with emphasis on the head and neck region. Hum Pathol 39: 633-640, 2008 .
4. Iida A, Imamura Y, Katayama K, Hirose K and Yamaguchi A: Plexiform schwannoma of the small intestine: Report of a case. Surg Today 33: 940-943, 2003.

5. Kawaguchi S, Yamamoto R, Yamamura M, Oyamada J, Sato H, Fuke $\mathrm{H}$ and Yabana T: Plexiform schwannoma of the rectum. Dig Endosc 26: 113-116, 2014.

6. Gkekas C, Kalyvas V, Symeonidis EN, Malioris A, Papathanasiou M, Kalinderis N, Moisidis K, Hatzimouratidis K: Plexiform schwannoma of the penis: A rare subtype of genital Schwannoma. Case Rep Urol 2019: 1752314, 2019.

7. Gangopadhyay AN, Sharma S, Kumar M and Sharma SP: Presacral schwannoma with intraspinal extension in a child with neurofibromatosis type 2-A case report and review of literature. J Indian Association Pediatric Surgeons 9: 116-119, 2004.

8. Aktekın A, Özkara S, Merıç K, Günay Gürleyık M, Aker F and Sağlam A: Plexiform schwannoma of the duodenum accompanying pyloric stenosis: Report of a case. Turk J Gastroenterol 23: 385-389, 2012.

9. Hirose T, Scheithauer BW and Sano T: Giant plexiform schwannoma: A report of two cases with soft tissue and visceral involvement. Mod Pathol 10: 1075-1081, 1997.

10. Shishiba T, Niimura M, Ohtsuka F and Tsuru N: Multiple cutaneous neurilemmomas as a skin manifestation of neurilemmomatosis. J Am Acad Dermatol 10: 744-754, 1984.

11. Retrosi G, Nanni L, Ricci R, Manzoni C and Pintus C: Plexiform schwannoma of the esophagus in a child with neurofibromatosis type 2. J Pediatr Surg 44: 1458-1461, 2009.

12. Ishida $\mathrm{T}$, Kuroda $\mathrm{M}$, Motoi $\mathrm{T}$, Oka $\mathrm{T}$, Imamura $\mathrm{T}$ and Machinami R: Phenotypic diversity of neurofibromatosis 2: Association with plexiform schwannoma. Histopathology 32: 264-270, 1998

13. Matsuoka Y, Kakudo N, Fukui M and Kusumoto K: Giant plexiform schwannoma in the plantar aspect of the foot: A case report. J Surg Case Rep 2019: rjz352, 2019.

14. Fisher MJ, Belzberg AJ, de Blank P, De Raedt T, Elefteriou F, Ferner RE, Giovannini M, Harris GJ, Kalamarides M, Karajannis MA, et al: 2016 Children's Tumor Foundation conference on neurofibromatosis type 1, neurofibromatosis type 2, and schwannomatosis. Am J Med Genet A 176: 1258-1269, 2018.

15. Karajannis MA, Legault G, Hagiwara M, Ballas MS, Brown K, Nusbaum AO, Hochman T, Goldberg JD, Koch KM, Golfinos JG, et al: Phase II trial of lapatinib in adult and pediatric patients with neurofibromatosis type 2 and progressive vestibular schwannomas. Neuro Oncol 14: 1163-1170, 2012.

16. Vitte J, Gao F, Coppola G, Judkins AR and Giovannini M: Timing of Smarcb1 and Nf2 inactivation determines schwannoma versus rhabdoid tumor development. Nat Commun 8: 300, 2017.

17. Broehm C, Al-Ibraheemi A and Fritchie KJ: Pediatric Non-vestibular Schwannoma. Pediatr Dev Pathol 20: 232-239, 2017.

18. Castellanos E, Plana A, Carrato C, Carrió M, Rosas I, Amilibia E, Roca-Ribas F, Hostalot C, Castillo A, Ros A, et al: Early genetic diagnosis of neurofibromatosis type 2 from skin plaque plexiform schwannomas in childhood. JAMA Dermatol 154: 341-346, 2018

19. Ijichi K, Muto M, Masaki A and Murakami S: Recurrent plexiform schwannoma involving the carotid canal. Auris Nasus Larynx 45: 358-361, 2018.

20. Sivak MV Jr, Sullivan BH Jr and Farmer RG: Neurogenic tumors of the small intestine. Review of the literature and report of a case with endoscopic removal. Gastroenterology 68: 374-380, 1975.

21. Agaram NP, Prakash S and Antonescu CR: Deep-seated plexiform schwannoma: A pathologic study of 16 cases and comparative analysis with the superficial variety. Am J Surg Pathol 29: 1042-1048, 2005. 